\title{
Change of the ovulatory function of the rats under the influence of the pharmacological blockade of melatonin receptors
}

\section{Oksana Valentinovna Zhukova ${ }^{1}$, Irina Anatolievna Vinogradova ${ }^{1}$, Elena Sergeevna Obukhova ${ }^{1}$, Eugene Aleksandrovich Khizhkin², Victor Aleksandrovich Ilyukha ${ }^{2}$}

${ }^{I}$ Petrozavodsk State University, Russia, ${ }^{2}$ Institute of Biology of the Karelian Research Center of the Russian Academy of Sciences, Petrozavodsk., Russia

The presence of melatonin receptors in the uterus and ovaries suggests that the melatoninergic system plays an important role in the regulation of the reproductive function. Luzindol is a selective blocker of type 1 and 2 melatonin receptors (MT1 and MT2) which disrupts the synchronization of circadian rhythms due to the action of melatonin.

Materials. Female rats born and left in conditions of light deprivation. In 5 months were divided into two experimental groups: 1st $(\mathrm{DD}+\mathrm{luz})$ the animals every day 5 days a week at night with drinking water received luzindole at a concentration of $10 \mathrm{mg} / \mathrm{l}$; 2nd (DD) rats were received at night drinking water. The third group (LD) control: animals were under standard mode lighting (12 h light/12 h night).

Parameters were estimated: duration of the estrous cycle; the relative number of short and medium and long cycles; the relative number of animals with irregular cycles.

Results. Determined that the average duration of the cycle with age increased: in the LD group from 4.8\&plusmn1.4 days to 7.4\&plusmn1.7 days; in the DD group from 4.4\&plusmn1.4 days to $5.3 \&$ plusmn1.8 days; in the DD+luz group from 4.4\&plusmn1.2 days to $7 \&$ plusmn1.4 days from 6 to 21 months respectively. In the LD group the number of long estrous cycles from 6 to 21 months increased from 3\% to 43\%; in the group DD+luz-from 2\% to 43\%. Short estrous cycles in these groups disappeared by 18 months. In the DD group the number of long estrous cycles increased slightly from $10 \%$ in 6 months to $20 \%$ by 21 months and short persisted in adulthood.

The first irregular cycles in the LD and DD+luz groups were established at 9 months by the 21st month they had increased to 33\% and 36\% respectively. In the DD group irregular cycles were recorded at 6 months by 21 months were $17 \%$.

Conclusions. Thus with the pharmacological blockade of melatonin receptors by luzindol at constant darkness the changes in the ovulatory function did not differ from the control group which indicates the determining role of melatonin receptors in regulating the age-related changes in the ovulatory function. 